\title{
Geospatial Semantics: Why, of What, and How?
}

\author{
Werner Kuhn \\ Institute for Geoinformatics \\ University of Münster \\ Robert-Koch-Str. 26-28 \\ D-48151 Münster (Germany) \\ kuhn@uni-muenster.de
}

\begin{abstract}
Why are notions like semantics and ontologies suddenly getting so much attention, within and outside geospatial information communities? The main reason lies in the componentization of Geographic Information Systems (GIS) into services, which are supposed to interoperate within and across these communities. Consequently, I look at geospatial semantics in the context of semantic interoperability. The paper clarifies the relevant notion of semantics and shows what parts of geospatial information need to receive semantic specifications in order to achieve interoperability. No attempt at a survey of approaches to provide semantics is made, but a framework for solving interoperability problems is proposed in the form of semantic reference systems. Particular emphasis is put on the need and possible ways to ground geospatial semantics in physical processes and measurements.
\end{abstract}

\section{Introduction: Why Semantics?}

In some sense, Geographic Information Systems (GIS) have always been based on semantics, sometimes even on explicitly defined semantics. For example, a GIS user in an environmental planning agency in Germany is likely to keep a heavy binder on her shelf. It is called the ATKIS Object Catalogue ${ }^{1}$ and its role is to define the object classes and attributes occurring in topographic data, both syntactically and semantically. Similarly, land use and land cover databases have always been built according to some semantic classifications, such as the European CORINE standard [1]. So, what has changed, and what would it mean today for a GIS to be based on semantics?

The answer is that access to and use of geospatial information have radically changed in the past decade. Previously, the data processed by a GIS as well as its methods had resided locally and contained information that was sufficiently unambiguous in the respective information community [2]. Now, both data and methods may be retrieved and combined in an ad hoc way from anywhere in the world, escaping their local contexts. They contain attributes, data types, and operations with meanings that differ from those implied by locally-held catalogues and manuals. Since the semantics specified by these local resources is not machine-readable, it cannot be shared with other systems. Coping with this situation defines the challenges of semantic interoperability [3].

\footnotetext{
${ }^{1}$ http://www.atkis.de/dstinfo/dstinfo2.dst gliederung2?dst ver=dst
} 
The notion of semantic interoperability is hard to pin down, for several reasons: it is somewhat redundant, there is no accepted formal definition, there are no benchmarks or commonly agreed challenges, the role of humans in the process is unclear, and the acronym inflation around the semantic web obscures rather than highlights the deeper research issues. Clearly, semantic interoperability is the only useful form of interoperability. In the real world, it is hard to imagine two agents interoperating successfully without a shared understanding of the messages they exchange. Therefore, it seems appropriate to define interoperability in a way that involves shared conceptualizations.

The following definition of interoperability that emerged from a geospatial context is often quoted (ISO TC204, document N271):

"The ability of systems to provide services to and accept services from other systems and to use the services so exchanged to enable them to operate effectively together."

This definition is almost identical to the one in Wikipedia². Such definitions are technical enough to be useful in systems engineering and testing. They also make clear that interoperability rests on services. But they fall short of establishing verifiable criteria. What does it mean for systems to operate together? And when can they be said to do this effectively?

A more precise definition of interoperability would require at least two steps: (1) identifying the vocabulary and syntax of service interfaces, and (2) defining interoperability mathematically. In this paper, I address the first requirement. Preliminary results of an ongoing debate ${ }^{3}$ suggest that the theory of institutions $[4,5]$, building on category theory, supplies the necessary formal foundations for the second requirement.

Semantic interoperability is the technical analogue to human communication and cooperation. It hardly constitutes a research topic per se for Geographic Information Science, but serves as a technical goal justifying the formalization of semantics in GIS and providing measurable criteria of success for this undertaking. The research questions it raises range from those of ethnophysiography, which studies how people conceptualize landscape features, to questions about human cognition of geospatial information in general $[6,7]$, through formalization methods for geospatial concepts [8] and architectures for ontology-based GIS [9], to socio-economic aspects of spatial data infrastructures [10].

The notion of interoperability needs to be understood broadly enough, encompassing the interoperation between human beings and systems. But it should also remain precise enough, allowing for a common syntactic basis. While it is essential to consider the organizational and societal issues involved in information sharing [11], it is detrimental to overload the definition of technical interoperability with these aspects. Levels of interoperability should be defined incrementally, starting at the purely technical and proceeding through the organizational and social levels. Sooner rather than later, however, environments for semantic interoperability will have to include means for meaning negotiation and other ways of dealing with organizational and social contexts [12].

\footnotetext{
2 http://en.wikipedia.org/wiki/Interoperability

${ }^{3}$ http://www.dagstuhl.de/04391/Materials/
} 
The Muenster Semantic Interoperability Lab (MUSIL ${ }^{4}$ ), as well as other research groups (see, for example, [13]), have found that a focus on actual interoperability problems helps to sharpen the research questions around the broad theme of semantics of geospatial information. Investigating interoperability scenarios based on actual cases of using geospatial information for decision making provides measures of success to test specific semantic and technological hypotheses: a certain choice of concepts specified in an ontology, or certain elements in a service architecture should produce a difference in the degree of interoperability between some components. With a formal definition of interoperability, the difference could even be measured.

This paper shows what syntactic parts of geospatial information need to be specified semantically to support interoperability (Section 2); it classifies semantic interoperability problems and illustrates them through scenarios (Section 3); it postulates a solution framework inspired by spatial reference systems (Section 4), and concludes with a summary and an outlook on longer term research challenges (Section 5).

\section{Semantics of What?}

This section defines the bases for semantic interoperability research by asking "what needs to be semantically specified in order to support semantic interoperability?" It clarifies the notion of semantics and the syntax of the expressions which require semantics to achieve interoperability. The fundamental construct of a service interface is highlighted and analyzed. Since the perspective taken on semantic interoperability includes human beings as parts of interoperating systems, user interfaces are subsumed under service interfaces. Finally, the question "what is special about spatial" is revisited in the context of geospatial semantics.

\subsection{Semantics}

The only sensible use of the term "semantics" refers to the meaning of expressions in a language. Such expressions can be single symbols (the "words" of a language) or symbol combinations. As the term implies, they are used to express something, i.e., to communicate meaning. Neither concepts nor entities nor properties nor processes have semantics, but expressions in languages describing them do.

The relevant languages in an information system context express how human beings conceptualize something for the purpose of representing and manipulating it in machines. Many such languages exist and need semantics: programming languages, schema languages, query languages, interface specification languages, workflow modeling languages, user interface languages, sensor modeling languages, and others. Many of these languages allow users to define new symbols (for individuals, types, properties, relationships etc.). Additionally, application standards introduce all sorts of more or less controlled vocabularies (such as those in feature-attribute catalogues or metadata standards). Furthermore, free-form text entries in data and metadata col-

${ }^{4}$ http://musil.uni-muenster.de 
lections open the gate to almost unlimited uses of natural language expressions. Coping with the semantics of all expressions in such languages is beyond current means. Restricting the expressions to those affecting interoperability will make the task more manageable.

Attaching meaning to language expressions is a conceptual phenomenon. Natural language symbols and expressions evoke concepts in human minds and are used to express those concepts. For example, the term "jaguar" can evoke a concept of an animal, car, or jet fighter in a human mind, with context usually picking out the intended interpretation and discarding the others. The concepts, in turn, are shaped by human experience with some real-world entities. Thereby, expressions come to refer to entities (as well as properties, relationships, and processes) in the world. This fundamental ternary meaning relationship between symbols, concepts, and entities is captured in the so-called semantic (or semiotic) triangle, going back at least to [14], but already implicit in Aristotle's work. The triangle exists in many versions; the one shown here considers the three relationships forming the edges as human activities (using a symbol to express a conceptualization of something in the real world, and to refer to that):

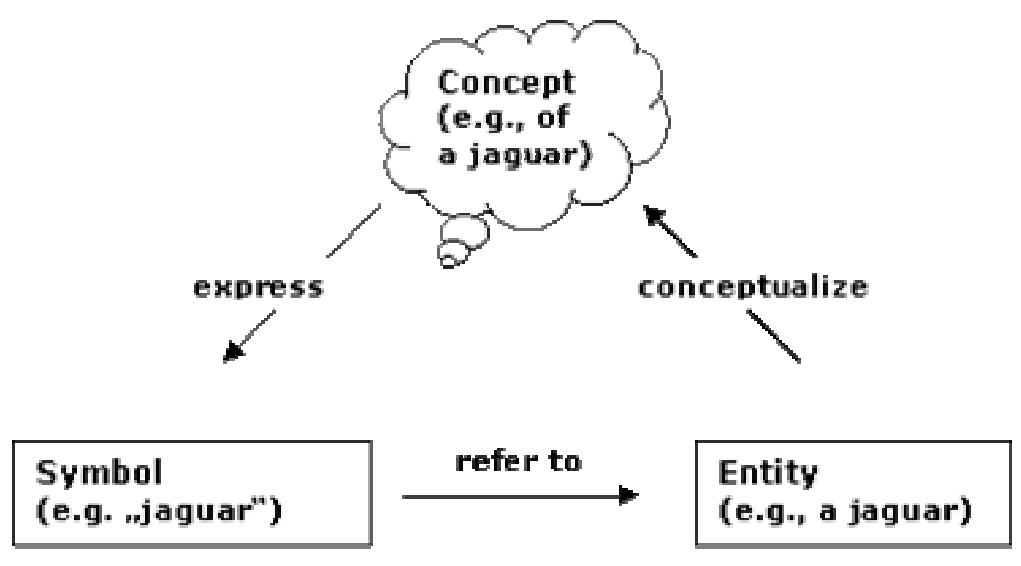

Fig. 1. The semantic triangle

The languages used in information systems are not natural languages, even if they use natural language terms. They are the results of social agreements in information communities on how to use certain terms; agreements which are typically more explicit than those underlying the use of natural languages. The agreements establish technical terms (say, overlap as a topological operator), which are recognized to have a relatively fixed meaning that is sometimes formally defined and often made explicit in the form of feature-attribute catalogues, interoperability standards, legal regulations, and other defining documents. For example, the navigation community has agreed on various forms of graph representations to model road networks for navigation purposes [15]. Codifying such agreements in ontologies is a useful first step toward semantic interoperability $[16,17]$. 
The symbols and expressions of information system languages can be produced or consumed by machines, but acquire meaning by the same relationships as those of natural languages. The fundamental fact about meaning, that it is generated by humans and not defined by a state of the world, applies to all symbols, and independently of whether they stand for individuals (as names or constants do) or classes (as nouns or type labels do). This view of semantics avoids the pitfalls of simplistic associations between symbols and entities in the world, sometimes referred to as realist semantics [18].

Geospatial semantics, consequently, is not about the relationship between GIS contents and the world, and does not need to be: this relationship is already captured in the notion of correctness (and, more generally, integrity) of databases and information systems. Geospatial semantics is about understanding GIS contents, and capturing this understanding in formal theories. At the same time, one should not make simplistic assumptions about the nature of the concepts that define such understanding. They are not just individual notions, but constantly evolving and often elusive results of conceptualization processes in information communities.

Is the goal of research on geospatial semantics to fully specify the semantics of geospatial terms? Such an enterprise would be too daunting, but also unnecessary. Consider how well human communication works without precisely defined semantics. We all use one or more natural languages (such as English or Mandarin) to communicate, none of which has a formally defined semantics. Yet, we understand and cooperate with each other reasonably well, despite frequent semantic ambiguities. As human beings living in certain social contexts, we have devised means of resolving these ambiguities as far as necessary to make communication and cooperation successful. This fact should caution us against putting more emphasis on formalizing meaning than on the reasoning that uses these formalizations to make necessary distinctions. Nevertheless, a few words on formalization are in order before addressing the reasoning challenges posed by interoperability.

\subsection{Formalizing Semantics}

Since concepts (and meanings, as relationships between expressions, concepts, and the world) are not directly observable, theories of semantics have to introduce substitutes for them. They can choose to represent meaning as a relationship between symbols (symbols of a language and symbols representing concepts) or instead represent effects of meaning (for example, the actions in the world resulting from understanding an expression). The former option is taken by the field of formal semantics and constitutes the only practical approach today. The latter requires theories of action (and of the role of information in them) that are not available yet for geospatial applications. As it would compensate some shortcomings of formal semantics, I will discuss this option in some more detail in Section 4.

Formal semantics, as coming out of logic, linguistics, and computer science, establishes a mathematical basis to talk about meaning. Through model theory, it introduces the notion of possible models, formally defining the semantics of expressions [19]. These models are considered to be the meanings. From a conceptual point of 
view, they are just symbolic structures, albeit useful ones: They represent conceptualizations of entities, properties, and relationships in a domain and can therefore be tested against human intuitions about these [20]. Differences observed between the intuitions and the behavior of the models can then suggest possible changes to the models.

Thus, model theory allows, at least in principle, for empirical tests of hypotheses about the semantics of expressions. Such hypotheses are formulated in information system ontologies. An ontology is a "logical theory accounting for the intended meaning of a formal vocabulary" [21]. It has to be logically satisfied by its models. The closer the models correspond to the human concepts about a domain, the more useful will an ontology be. The richer the models are, the more powerful hypotheses can be tested. But the questions how detailed and how expressive ontologies should be are purely pragmatic ones. The answers depend on the levels of interoperability to be supported.

The perceived limitations of model-theoretic approaches to meaning are largely due to a limited notion of models, which are often restricted to sets. Unstructured sets are almost always too weak to serve as interesting conceptualizations of the world. For example, they cannot adequately model mereological relationships, which are essential for spatio-temporal applications [22]. Humans do not understand domains as sets of things and subsets formed by predicates, but through their behavior and the actions that can be performed in them [23]. A road is a road by virtue of linking places in a way affording cars to drive from one place to another. A lake is a lake because it holds standing water and serves as a (possibly empty or frozen) water reservoir, for swimming, sailing, and even driving [24]. Modeling such processes through operations creates an algebraic structure, which captures meaning through models and associated mappings (morphisms) within and across domains [25].

A more fundamental pitfall of model theory (and of any other theories of meaning based on symbolic structures) lies in the symbol grounding problem [26]: how do the language (and model) symbols acquire meaning? Describing their meaning by other symbols begs this question. Languages are much richer systems than formal symbol systems. The meaning of their expressions emerges, lives, and evolves in language users and communities, where human bodies and minds communicate [27]. Meanings are not fixed and cannot be assigned to symbols independently of how these are used. All symbolic approaches to semantics, therefore, are necessarily limited in scope and need to be complemented by studies of language use and evolution [28]. Breaking out of the symbolic cage will eventually require pursuing the option of accounting for meaning by modeling observable effects in the world (see Section 4).

\subsection{Semantics of Services}

The focus on semantic interoperability picks out a small subset of languages and defines their semantics: those used to specify and invoke services. Consequently, the semantics required to achieve interoperability is that of expressions built from symbols in service descriptions. In the semantic web context, various languages are used to write such expressions. For example, WSDL (the Web Service Description Lan- 
guage) allows for syntactic descriptions of web service interfaces and OWL-S (the service ontology of the Web Ontology Language) has been proposed for semantic specifications of services. More comprehensive service modeling efforts like WSMF (the Web Service Modeling Framework) are under way [29].

It is not always clear how service descriptions are to obtain semantics and what additional modeling languages may be needed. In particular, it is difficult to say something meaningful and useful about the operations performed by services. The semantic web approach is to specify input and output types, pre- and post-conditions, and taxonomies of service types. But the form and use of pre- and post-conditions are unclear, the granularity of service taxonomies is too coarse, the service types themselves are not semantically defined, and the algebraic structure that operations impose on domains cannot be captured. We simply do not know yet what needs to be said, and how, about the semantics of services to make them semantically interoperable. It makes sense, therefore, to take a step back and study their vocabulary and syntax in more detail.

\subsection{Interfaces}

Agents, computational and human, interoperate through interfaces. For meaningful and useful interoperation, these interfaces need well-defined semantics. In today's GIS service architectures, the interfaces are those of web services, interacting with each other or with human users. The success of the transition from the distributed computing platforms of the nineties and earlier architectures to web-based service architectures depends to a large extent on the development of techniques to specify and query the semantics of service interfaces.

The idea of an interface is well understood in computer science and provides an excellent basis for modeling its semantics. For more than thirty years, software engineers have known that the semantics of data and operations are inseparable and that they are best explained in terms of interfaces of software components [30]. Based on this insight, the Open Geospatial Consortium $\left(\mathrm{OGC}^{5}\right)$ has transformed the architecture of GIS over the past decade. Acknowledging the role of interfaces for distributed computing [31] in the GIS area, OGC identified the interface of software components as the key ingredient of GIS technology that needed standardization in order to achieve interoperability [32]. The result is a series of syntactic interface specifications, establishing protocols for components exchanging geospatial information. The information so exchanged can contain features, maps, coverages, or metadata.

These service interface standards establish syntactic interfaces and protocols for invoking system behavior, but do not specify the intended meaning of their terms in machine-readable form. Consequently, individual components can only be tested for conformance to specifications, but not for interoperability with each other. The need to attach semantics to the syntax specifications has been well recognized in OGC from its early days. It led to the vision of services interoperating within and across information communities through semantic translation [33].

\footnotetext{
${ }^{5}$ http://www.opengeospatial.org
} 
From a semantics point of view, one wishes that OGC's focus on interfaces had not been diluted by the recent shift of attention to data exchange through GML (the Geography Markup Language). GML is a very useful and necessary schema language, but major efforts are now going into the exposure and harmonization of database schemata, activities which used to be considered unnecessary and even dangerous (because they lock providers and users into fixed data models). To bridge between the original vision and the current practice, the oxymoron of "data interoperability" has crept into industry and agency jargon. It suggests, figuratively speaking, that the flower and eggs in your kitchen interoperate among themselves to prepare pancakes. The point is not that humans need to be involved (this may or may not be the case), but that it takes operations to interoperate, not just data [34].

The user interfaces of software systems share many properties of component interfaces: they contain commands with well-defined syntax and observable semantic properties. Input parameters are being set, operations get executed, and results are returned. Thus, user interfaces exhibit, at least in principle, the same syntactic structure and behavior as internal component interfaces. Their input and output types are often more complex, but form interfaces of the same kind as those of object classes. These interfaces are equally or more important in the quest for semantic interoperability than internal interfaces, because, ultimately, information is always from and for human beings. Users are essential parts of interoperating systems.

\subsection{Signatures}

The interface of a service is formally captured by its signature. A signature describes a service's type information, consisting of the input types, output types and names of the operations offered by the service. Without loss of generality, I assume here that a service consists of a single operation. A core geospatial example of a service is the specification of the distance operation in the ISO Spatial Schema standard [35]:

$$
\text { GM_Object: : distance (geometry: GM_Object): Distance }
$$

This signature says that the distance operation shall be applied to a geometric object (GM_Object), takes as input another such object (called geometry), and returns a value of type Distance. The two geometries could be points or other geometric objects.

The notion of a signature is fundamental to algebra and category theory [36] and plays a central role in algebraic software specifications [37, 38]. Signatures contain symbols expressing

- values

- objects

- functions.

All these symbols stand for either an individual or a type. In the distance example, GM_Object expresses a type of object, distance an individual function, geometry an individual object, Distance a type of value. A call to a service implementing the distance operation would return an individual value. Note that the semantic characteristic of a measuring unit is normally considered to be a part of the 
function representing the measurement. Strictly speaking, an individual function implies a particular choice of unit (such as meters). For example, the interpretation of a result value 100 as 100 Meters would be given by the particular distance function.

Taken together, the type symbols in the signature describe the type of the distance function. The standard mathematical form of the signature shows this more clearly:

distance : GM_Object x GM_Object • Distance

It says that distance is a function of type GM_Object ${ }^{2} \rightarrow$ Distance and treats the two geometries symmetrically, as one would expect from a distance function. A shortcoming of today's service specification and implementation languages is that they rarely allow for this decoupling of functions from single value or object types.

Thus, service signatures as a whole, and the symbols they contain, express conceptualizations of a domain in terms of values, objects, and functions. Functions can have any number of arguments, including none, in which case they are individual values (such as True and False) or objects. The following table summarizes the kinds of concepts expressed by service signatures, with each cell showing an example from the distance service:

\begin{tabular}{|l|l|l|l|}
\hline Concept & Value & Object & Function \\
\hline Individual & 100 & Point_A & distance \\
\hline Type & Distance & GM_Object & GM_Object ${ }^{2} \rightarrow$ Distance \\
\hline
\end{tabular}

Table 1. Kinds of concepts expressed in service signatures

The different kinds of concepts are closely related to each other in two orthogonal ways:

- each individual (value, object or function) is an instance of a type;

- values and objects are the arguments and results of functions.

Our analysis of service interfaces has thus revealed a well-defined and relatively small set of semantic elements and relationships that need to be defined to enable semantic interoperability.

\subsection{Geospatial Semantics}

In the absence of a general theory of service semantics, it is hard to state clearly why and how geospatial services may be special. At the structural level of establishing semantics for service signatures, there does not seem to be anything special about space (or time). Yet, the geospatial data types and operations occurring in these signatures, and the conceptualizations underlying them, are characterized by some important properties (see also [39] for an implementation-oriented view):

1. Geospatial data and services contain symbols whose meaning is not only a matter of convention, but grounded in physical reality. For example, a wind direction returned by a weather service or a water level measured by a gauge have an observable grounding in the world. Conversely, the meaning of their measuring units, of 
a currency amount, or of a single-click purchase at an e-commerce site is purely conventional. Because of this physical grounding of some concepts, explaining the semantics of geospatial information requires measurement ontologies [40] that are tied to existing standards in science and engineering [41].

2. At the same time, geospatial information is often based on human perception and social agreements, combining objective measurements with subjective judgments. Coping with the meaning of qualitative judgments (say, of statements on landscape aesthetics) or of social constructions (like neighborhood classifications), and providing mappings among them [42], are probably the biggest challenges ahead to make geospatial information more meaningful and shareable. They require a layered architecture of ontologies [43], not just different unconnected perspectives or different levels of application specificity.

3. A special case of social agreements are geographic names and other identifiers of geospatial entities. Geographic name registries in the form of gazetteers will need better translation and geo-referencing capabilities. Object identifiers in different databases across information communities will need to be linked. For example, the same petrologic sample may be registered under different identifiers and referenced to different geographical names in various online databases supporting geochemical analyses [44].

4. Space and time are primarily understood through processes: we locate stuff because we can move it (not the other way round!), we use distances and directions to navigate, and we determine when to leave the beach by estimating the speed of an advancing storm. This process-nature of geospatial information challenges the entity-bias of the semantic web and geospatial data models [45], though the challenge as such is surely not unique to geospatial applications.

5. Geospatial ontologies can be seen as "GIS at the type level”. They should provide reasoning capabilities (spatial and non-spatial) about types of geospatial values, objects, and functions, similar to the reasoning provided by GIS about their instances. For example, to determine the feature types to be retrieved for flood risk assessment, one has to reason about spatial relations like proximity between entities and rivers, independently of particular feature instances.

6. Vagueness, uncertainty, and levels of granularity are fundamental to geospatial information. Theories of vagueness and uncertainty, as well as mappings among spatio-temporal [46, 47] and semantic [48] granularity levels of ontologies are therefore essential ingredients of geospatial semantic theories.

Clearly, this is an open-ended list of characteristics, and none of them applies only to geospatial information. But it is useful to keep such considerations in mind when evaluating approaches to semantic modeling for geospatial domains. Equally important, however, is to clarify the interoperability problems to be solved through the semantic models. These problems are the subject of the next section.

\section{A Classification of Semantic Interoperability Problems}

The premise that interoperability is the technical reason to model the semantics of geospatial information, together with the defining role of services for interoperability, 
allows for identifying and classifying interoperability problems. This section introduces three problem classes through geospatial examples. The problem classes are orthogonal and complementary to the types of semantic heterogeneities (naming, conceptual) discussed in the interoperability literature [49]. They capture specific reasoning challenges that arise in the course of making systems and services interoperable. The necessary reasoning is often referred to as matchmaking and is here briefly introduced before discussing the problem classes.

\subsection{Matchmaking for Interoperability}

Matchmaking is the fundamental procedure enabling semantic interoperability [50]. It is a reasoning process with the goal of deciding whether an information offer matches a request. The reasoning can be performed by humans or software or a combination of both. Its result can be binary (match or not) or a measure for the degree of match, i.e., for similarity.

The main task in matchmaking is to determine and resolve semantic heterogeneities between requests and offers. There are naming heterogeneities (different expressions for the same concept) or conceptual (a.k.a. cognitive) heterogeneities (different concepts expressed by the same symbols). The naming heterogeneities are sometimes further subdivided into syntactic (different symbols) and structural (different expressions). An example for a syntactic naming heterogeneity is a distance value expressed as a floating point number or as a distance type. A structural naming heterogeneity is that between a location expressed by two separate coordinates or by a point data type. A conceptual heterogeneity would be that between a distance computed on the sphere or in a plane.

Clearly, matching data to specifications and resolving the corresponding heterogeneities is much easier than matching services to specifications. These two cases define the first two classes of interoperability problems. An even more difficult case of matchmaking is the reasoning to determine whether and how services can be composed to produce a desired behavior. The matches sought are then between the services (to form a combined service) and between the composed service and the request. This case defines the third problem class. Each of the three problem classes includes the previous one as a part of the problem.

\subsection{Data Discovery and Evaluation}

Today, the bulk of digital geospatial information resides in databases and files. Users of these data need information on what they mean. No matter whether they access the data through web sites, database queries, import functions, connections to data warehouses, or OGC web services - at some point they will receive values, attribute names, and complex objects. Searching for data sources and evaluating their contents define the first class of semantic interoperability problems.

Consider a hydrologist searching for information on water levels of the river Elbe [51]. She may be in charge of issuing flood warnings or monitoring ecological indica- 
tors. Among the data sources at her disposal are gauge readings from different stations. Three examples of water level data providers on the World-Wide Web are:

- The German Federal Agency for Hydrology 6 ;

- The German Electronic Information System for Waterways;

- The Czech Hydrometeorological Institute ${ }^{8}$.

The data offered by these sources consist of attributes for station names and water levels, time stamps, station locations, river names, and additional hydrological information on water discharge and the like.

Interfaces to data represent the special case of (service) interfaces without computational functionality. Their structure can therefore be described by signatures, and the concepts expressed by the symbols are a subset of those in Table 1, leaving away its last column:

\begin{tabular}{|l|l|l|}
\hline Concept & Value & Object \\
\hline Individual & 158 & Elbe \\
\hline Type & Höhe & WasserstandMessung \\
\hline
\end{tabular}

Table 2. Kinds of concepts symbolized in data repositories

For example, the data source providing the value of 158 in Table 2 declares it to be of type Wasserstand for a given Pegel (station), Datum (date) and Uhrzeit (time). Even if the German terms could be interpreted by a client (human or software), the measuring unit, reference level, and measurement or averaging process for the water level remain unspecified.

The matchmaking needed to discover and evaluate data sources has to resolve such ambiguities. Existing metadata standards and catalog services do not support this process well [51]. Their contents and search procedures are keyword-based, similar or inferior to those of internet search engines, with no way of resolving naming or conceptual heterogeneities. The keywords are not treated as values of different types, and normally not taken from controlled vocabularies. They are just strings, to which machines cannot attach any meaning, and humans may or may not apply the right interpretation. So far, the main efforts in using the semantic web for geospatial applications have been geared to improve this situation [52].

\subsection{Service Discovery and Evaluation}

While only a relatively small amount of geospatial information is provided in service form today, the number and computational power of geospatial information services is growing rapidly. In addition to data access, such services offer processing and portrayal capabilities. They may be coupled to specific data sources or applicable to data

\footnotetext{
${ }^{6}$ http://www.bafg.de/php/elbe.htm

${ }^{7}$ http://www.elwis.de/gewaesserkunde/Wasserstaende/Wasserstaende_start.php? target=2 $\underline{\mathrm{g} w}=\mathrm{ELBE}$

${ }^{8}$ http://www.chmi.cz/hydro/SRCZ04.html
} 
from multiple sources. Discovering and evaluating such services represents the second class of interoperability problems.

The additional (and more challenging) semantic issues in this second problem class arise from the need to reason about the functionality of services. Describing the meaning of an operation like distance is far from trivial: the operation signature can refer to many different kinds of distances (metrics), ranging from the path length in a graph through the Euclidean or Manhattan distances in the plane to a geodesic or straight-line distance on the surface or across the earth [53]. All of these distance operations have the same signature shown above (i.e., they are of the same type).

Obviously, the functionalities of more complex geoprocessing operations (such as buffering or overlay, but also topological operators [54]) pose even harder semantic challenges. If the functionality descriptions become too complex, they are unlikely to be produced by service providers and understood by clients. But if they are too simple, recall and precision in discovery and evaluation are reduced. Furthermore, the descriptions need to support the reasoning necessary to match service offers to requests. If this reasoning becomes too expensive, it threatens the efficiency of service discovery and evaluation. Traditional specification methods from software engineering turn out to be either too weak in expressiveness or too complex for the available reasoning mechanisms [40]. Process ontologies seem a promising alternative [55], but their contents and associated reasoning methods are not yet clear, and they lack spatiotemporal notions [47].

The semantic heterogeneities in this second class of semantic interoperability problems concern all six kinds of symbols shown in Table 1. But peculiar to this problem class are the semantics of function types and individuals. For example, the type GM_Object ${ }^{2} \rightarrow$ Distance of a distance function needs an interpretation and so does the individual function symbol distance. The function type specified in the ISO Spatial Schema Standard is quite precise, with an explicit result type Distance. However, implementations typically use a more generic result type, such as a floating point number. The semantics of the function type (e.g., GM_Object ${ }^{2} \rightarrow \mathrm{Fl}$ oat) then becomes highly ambiguous and can be interpreted as any real-valued property of two geometric objects. As a consequence, the full semantic burden rests on specifying the individual function identifier, distance.

Some semantic heterogeneities in service signatures can be resolved through spatial reference systems [56]. These provide information about the spaces in which the arguments of an operation (the geometric objects) are embedded. For example, the spatial reference system of two geometric objects going into a distance operation may be a plane coordinate system tied to a certain map projection. One can safely assume that a distance should be calculated in that same reference system, though this still leaves open which metric it uses. Also, a distance service that is decoupled from a data source would either need to be restricted to a fixed reference system and metric, with service metadata describing these choices, or carry the generic and complex functionality for all possible combinations. Finally, the case of the two geometric objects having different reference systems needs to be resolved (as, for example, in [35]: "If necessary, the second geometric object shall be transformed into the same coordinate reference system as the first before the distance is calculated").

Current GIS practice does not suffer much from this second class of interoperability problems. It uses coarse-grained generic service interfaces, like those of feature 
servers, and combines them with database schema exchange through GML. Feature, coverage, and map services as specified by OGC essentially provide semanticsneutral wrappings for repositories of vector, raster, and map data. This puts us back to problem class one. Admittedly, the idea of finer-grained service interfaces, which used to be seen as the core of interoperability in the geospatial area, has been hampered by complexities like those exhibited in CORBA applications [57]. But by breaching the information-hiding principle of object orientation and exposing internal data formats, data get separated from the operations they were created for (or by), and a heavier price has to be paid to restore meaning to them. The general evolution toward finer-grained functionality offered over the web may bring the second class of interoperability problems to the forefront again.

\subsection{Service Composition}

Full-fledged semantic interoperability involves not just individual services to be discovered and used, but multiple services interoperating with each other. The third semantic interoperability problem class is defined by the semantic issues raised through automated or manual compositions of services to produce more complex services or entire applications. It is characterized by the need for these services and their clients to share an understanding of what the services do and what goes across their interfaces [58].

Consider a service to compute the outline of a toxic cloud at some point in time after a chemical accident, taking as inputs a report on the chemical accident and data from a weather service [59]. Assume that the accident report provides location, time, type and emission rate of chemical, while the weather service returns wind direction and speed. The values, objects, and functions involved pose the same kinds of semantic heterogeneity issues as in problem class one. For example, the functionality of the plume calculation service uses a certain spatio-temporal resolution and a threshold concentration of the chemical to determine the outline.

Matchmaking in this problem class, however, is more complex. Service requests may depend on previously found offers for other services. This interdependency leads to a more involved reasoning process, spanning over requests and offers of multiple services. For example, the wind information supplied by the weather service may differ from the one expected by the plume service (e.g., it may follow the meteorological standard of westerly wind blowing from the west, while the plume service might expect a vector direction, such that a $270^{\circ}$ wind blows to the west). The request for weather information, then, depends on the plume calculation.

Furthermore, this problem class involves mediation between the output provided by one or more services and the inputs required by another. For example, a semantic translation from one conceptualization of wind direction to the other may be needed. This translation task remains the biggest challenge of semantic interoperability, particularly when it concerns service functionality. 


\section{A Framework for Solving Semantic Interoperability Problems}

What methodological approach is required to solve the semantic interoperability problems defined in the previous section? All three problem classes have been characterized as involving matchmaking, i.e., reasoning about the compatibility of offers and requests for data or services. This reasoning perspective emphasizes the need for approaches that go beyond the construction of ontologies and involve their use for discovering, evaluating, and combining geospatial information. Semantics-based GIS are about reasoning, not just about ontologies. This section presents some thoughts on the reasoning requirements and a methodological framework in the form of semantic reference systems.

\subsection{A Geospatial Analogy}

One can think of ontologies as establishing conceptual “coordinate systems", i.e., frames of reference for positioning concepts in a certain context. For example, the concept of a car can be specified in an ontology as a specialization of a vehicle. If vehicles are in turn specializations of conveyances, one can conclude that cars are special conveyances, sharing all their properties and relationships. This taxonomic reasoning is fundamental to most ontology applications today. It has a spatial analogue in (set) containment: all instances of cars are contained in the set of all conveyances.

Taxonomic reasoning is useful, but insufficient for the matchmaking tasks described above. Equally or more important are non-taxonomic relationships, e.g., that wind direction and speed are parts of a wind force, or that a car can move on roads. Reasoning with these is much harder, as it is not of the simple set inclusion kind required for taxonomies, but depends on the semantics of each relationship. For example, complex relationships between moving air masses, locations, and measurement scales define a concept like wind direction [59].

Coordinate systems in geometric spaces allow for computing distances. Conceptual coordinate systems should support the computation of conceptual distances and similarities based on these. Similarity theories exist, also for geospatial concepts [60], and show the importance of capturing the context-dependence of human similarity judgments. Several approaches exist to cope with this problem. They are, for example, based on modeling the use of entities [61] or on "factoring out" context through relative similarities ( $a$ is more similar to $b$ than to $c$, or a is more similar to $b$ than $c$ is to d). In addition to context, all similarity theories are challenged by the question whether they should compare individuals, types, individuals to prototypes, or any combinations of these.

Context is an overloaded term and has many aspects. Some of them are relatively easy to handle through domain separation (e.g., the difference between banks in a financial and in an ecological context). Others are much harder to deal with, because they result from different groundings for the meaning of a symbol. For example, the difference between a mathematical (blowing to) and meteorological (blowing from) conceptualization of wind direction has a physical grounding. As long as such groundings are not represented in ontologies, no amount of taxonomic, non- 
taxonomic, or similarity reasoning can distinguish them or even reconcile their differences. Today's ontologies are islands in a sea of different conceptualizations, which are hard to connect [62]. They lack the means to ground conceptualizations in reality and therefore cannot solve the symbol grounding problem [26]: they do not anchor their conceptualizations in reality.

Geometric coordinate systems, by contrast, are anchored in physical features, such as fundamental geodetic points (materialized in monuments) and the rotation axis and parameters of the earth. This anchoring is called a geodetic datum [56]. The use of any coordinate system, spatial or otherwise, without anchors in reality is limited to local reasoning and cannot explain how the "coordinated" ideas relate to the world. Due to its lack of grounding, ontological reasoning today derives mostly local containment relations between the extensions of concept specifications. These are neither invariant nor translatable across multiple ontologies.

This rather loose analogy between meaning and geometry can be made stronger in several ways. Fabrikant, for example, is exploiting it for information access and visualization [63]. Gärdenfors has taken the idea of representing concepts geometrically very literally, in his notion of conceptual spaces [18]. His theory provides a solid mathematical basis for the analogy between concepts and geometric spaces and exploits it fruitfully for all sorts of representation and reasoning challenges, in particular for similarity measurements and transformations. Related in spirit, but with more emphasis on computational processes and less detail on representations, I have proposed the notion of semantic reference systems [64]. It takes the spatial analogy seriously in terms of the reasoning requirements it implies, and derives these from the computational power afforded by spatial reference systems.

\subsection{Semantic Reference Systems}

The information provided by a GIS is only useful if it rests on a well-defined spatial reference system. For example, way-finding directions refer to landmarks in reality and use distance and direction measurements anchored in physics. Maps represent the territory in a certain map projection, which allows for calculating distances and directions. Latitudes and longitudes can be traced back to arbitrarily exact locations on the surface of the earth. The meaning of a coordinate in a GIS database is entirely specified through the associated spatial reference system, and the meaning of geometric computations (such as distances) can be tied to the same system. The same is true for temporal data and reference systems. In other words, for coordinates and time stamps, we already have theories "accounting for the intended meaning of a formal vocabulary” [21], though they are based on algebra rather than logic.

Would it not be nice to have an equally powerful method of disambiguating the meaning of the remaining symbols carrying geospatial information, such as "wind direction” or “water level”? As Chrisman has already suggested [65], users of geospatial information should be able to refer thematic data to attribute reference systems, just as they refer geometric data to spatial reference systems. This idea suggests that the symmetry between the two components of Goodchild's geographic reality (a spa- 
tio-temporal location vector and an attribute vector [66]) is incomplete without reference systems for the attributes.

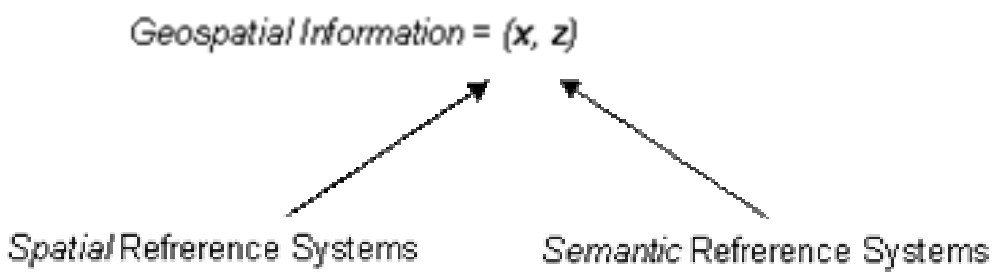

Fig. 2. Reference systems for interpreting geospatial information

From a semantic interoperability perspective, this requirement extends beyond attributes to cover all parts of service interfaces introduced in Section 2. I have therefore called for semantic reference systems to offer the necessary representations and reasoning capabilities for

- referencing symbols to concept specifications

- grounding concept specifications in physical reality

- projections among the semantic spaces

- transformations among different semantic reference systems.

While referencing is handled by ontologies today, grounding is not, projections are limited to piecewise generalizations in taxonomies, and translations need to make strong assumptions about shared conceptualizations. If computational solutions to these challenges appear unlikely, imagine the situation for theories of space as it was before Descartes invented coordinate systems. Sharing information about location, shape, and extent of things in the world was then probably just as difficult as sharing of non-geometric information is today. Fortunately, a solid mathematical theory of coordinate systems and their physical grounding is now available and can serve as a model for semantic theories and the capabilities they need to offer. Indeed, the best way to look at spatial reference systems is as the special (geometric) cases of semantic reference systems.

The representational, computational [67] and institutional challenges posed by this vision are substantial. However, they will need to be met, if the promise of semantic interoperability through the semantic web is to be fulfilled: a web “...in which information is given well-defined meaning, better enabling computers and people to work in cooperation” [68]. A formalization of Gärdenfors' conceptual spaces is likely to play a key role in implementing the reasoning capabilities, particularly projections and transformations [69].

Since the toughest challenge lies in the need for grounding, the rest of this section sketches two promising directions to pursue for grounding geospatial information in reality: based on image schemas and on measurements. The two ideas are connected through the key insight that image schemas are abstractions from experiences with processes in the world, which in turn have observable effects. Their emphasis on the 
links between processes, observations, and information is characteristic for a range of recent work related to geospatial information (see, for example, $[8,70,71]$ ).

\subsection{Grounding in Image Schemas}

Grounding the meaning of symbols through symbols is an oxymoron. Yet, some symbols (or symbolic structures) are more easily grounded than others. In other words, human interpretations of them are more likely to agree across domains and cultures through a shared understanding rooted in some physical processes. The claim behind the idea of an image-schematic grounding of ontologies is that symbolic structures representing image schemas possess this property.

Image schemas, as introduced by cognitive linguists and philosophers like Len Talmy, Ron Langacker, George Lakoff, and Mark Johnson (see [72] for a recent survey) are mental patterns shaping our thought, action, and language. They are rooted in our bodily and cultural experiences and extract the common structure of these. For example, the container image schema abstracts from our experience of dealing with and reasoning about anything that can contain anything else, such as cups, human bodies, or rooms. This experience is characterized by the processes of putting something into a container, discovering that it is inside, and taking it out again. Similarly, we build patterns from our experiences with surfaces, paths, links, covers, parts and wholes, centers and peripheries, force feedback, and an open-ended series of other structures (see p. 126 of [73] for a list).

As the examples show, many image schemas are spatial (mostly topological), and our understanding of them is process-driven, with an algebraic structure linking the processes (e.g., what is taken out of a container has been put into it before). The spatio-temporal nature and process character of image schemas, together with their claimed universality across languages and their cognitively fundamental role, predispose them as candidates for grounding the meaning of symbols in experiences of physical reality. Furthermore, image schemas are typically combined to generate more complex patterns, and transformed to emphasize certain parts. Finally, they have long been suspected to define those relationships that remain invariant under all sorts of semantic mappings, such as metaphor, translation, and conceptual blending [74].

For a geospatial semantics example, consider how to capture the concept of a road. One can do this in conventional taxonomic fashion by sub-classing it from a concept like construction (which in turn is sub-classed from artifact, physical object, etc.). With sophisticated ontologies (such as DOLCE ${ }^{9}$ ), one can even add constraints on a driving activity involving roads and vehicles. But this approach assumes that the upper levels of these ontologies are unambiguously understandable across domains. It also lacks the expressiveness to differentiate multiple ways for an entity (such as vehicle vs. a road) to participate in a process (such as driving).

Alternatively, one can introduce upper levels that represent image-schematic concepts, such as paths, containers, and surfaces. A combination of paths with containers, such that a vehicle is a container moving on a path (i.e., acting as a conveyance), re-

${ }^{9}$ http://www.loa-cnr.it/DOLCE.html 
sults in rich semantics for all participating concepts (vehicle, road, driving), while keeping the concept hierarchies flat. This shows that image schemas capture essential behavior of entities and provide useful grounding in our physical experience (of moving and containing, in this case). However, the question remains of how an image schema should be represented in order to evoke the intended interpretations. Still, in a sophisticated model theoretic view (see Section 2), this question can be answered empirically. Such ideas are currently pursued in the SeReS project ${ }^{10}$.

\subsection{Grounding in Measurements}

Geospatial information serves to understand the human environment and to decide on actions in it. One of its most important sources will soon be sensor networks. Despite the fact that many GIS applications produce and use information that remains valid for some time (such as geological or land cover maps), more and more decisions in organizations and societies rely on timely observations of the environment, and often almost real-time data (e.g., about traffic conditions). The ground-breaking technology of sensor networks allows for supporting such decisions in entirely novel ways. For example, vehicle navigation systems can access sensor data from stations or from vehicles ahead and alert drivers of conditions regarding weather, congestions, accidents, construction sites, and the like. Similarly, decisions on human activities affecting the environment will be supported by more current, higher resolution, and more relevant environmental data.

A quantum leap for information processing and interoperability will result from the possibility to insert geospatial information into feedback loops, where an activity is guided by observations on the effects of previous actions. For example, water level readings from a network of gauges in a river basin could be combined with hydrological models to guide preventive and corrective action in a flooding situation, and the results of these actions will become directly observable through the same network. International regulations (such as the European Water Framework Directive ${ }^{11}$ or the INSPIRE ${ }^{12}$ project targeting a European environmental information infrastructure) are now creating government mandates to collect and disseminate such information. Similar regulations can be expected in the security and health sectors.

These exciting technological and social developments add further semantic challenges to cope with, but also suggest a novel approach to grounding: All information ultimately rests on observations, whose semantics is physically grounded in processes and mathematically well understood [75]. Exploiting this foundation to understand the semantics of information derived from observations would produce more powerful semantic models. For example, a service interface consuming weather information could refer to a standard library of meteorological measurement types with welldefined semantics.

\footnotetext{
${ }^{10} \mathrm{http}: / /$ musil.uni-muenster.de/index.php?m1=Research\&m2=Semantic\&m3=Summary

11 http://europa.eu.int/comm/environment/water/water-framework/index en.html

12 http://inspire.jrc.it/home.html
} 
The Open Geospatial Consortium (OGC) has recognized the huge potential of these developments and created the Sensor Web Enablement initiative (SWE ${ }^{13}$ ). It proposes the special feature type of a "sensor observation" for comprehensive measurement data. Measuring units are to be dealt with through reference systems, which are nuclei (and precursors) for semantic reference systems. Their role is to define the context for interpreting measured values and to constrain the valid operations on them. This is, of course, a modern technological manifestation of Stevens' theory of measurement scales [75].

\section{Conclusions and Outlook}

In this paper, I have looked at geospatial semantics from the perspective of semantic interoperability. I argued that interoperability is the raison d'être for semantics research, and that it makes problems and hypotheses more specific and easier to test than a general "semantic studies" approach to geospatial information. I have treated semantics as a conceptual phenomenon, involving language expressions and human concepts, rather than as a correspondence between terms and situations in the world. But I have also stressed the need to anchor concept specifications in reality.

Against this background, I have asked what needs to be semantically defined in order to support semantic interoperability. The answer was that it is expressions built from service signatures, which can be considered the syntax of interoperability. Three classes of semantic interoperability problems were defined and discussed with respect to their reasoning challenges: data discovery and evaluation, service discovery and evaluation, and service composition. State-of-the-art solutions address mainly the first class of problems, while service semantics remains elusive, both for discovery and composition. Thus, I concluded that more powerful techniques than today's ontologies and reasoning environments are required to support semantic interoperability.

To serve this goal, I presented a framework for referencing, grounding, and mapping geospatial information in the form of semantic reference systems. The idea of such systems has been inspired by spatial reference systems and is intended to lead to analogue capabilities for non-coordinate symbols. Referencing is mostly addressed through the work on geospatial ontologies today. Grounding has been found to be particularly relevant for geospatial applications. As it has received little attention in the ontology literature so far, I sketched two complementary approaches to it: anchoring concept specifications in image schemas and in measurements. Mappings, in the form of projections and transformations between ontological specifications, will require such grounding and present the next major research frontier.

A theory of semantic translation, capable of mapping geospatial information within and across the boundaries of information communities, should indeed be seen as the overall goal of research on geospatial semantics. In the spirit of the geometric analogies used here and elsewhere [18], one can speculate that such a theory might take the form of an "Erlangen program" of meaning: a formalization of semantics based on invariants under certain groups of transformations, very similar to Klein's seminal

${ }^{13}$ http://www.opengeospatial.org/functional/?page $=$ swe 
work that put geometry on modern mathematical foundations (and to some extent helped create these foundations) in the second half of the $19^{\text {th }}$ century [76]. To connect such a theory to reality, the meaning of symbols used in geospatial information will eventually need to be tied to an understanding of how information supports decisions on actions, and how observations of the effects of actions in turn generate new information.

The big practical challenges ahead lie in an evolution from semantic interoperability to semantic integration of geospatial information. All interoperability problems are also integration problems. In order for two system components to interoperate, they must share an integrated view of some information contents. Information integration, however, goes far beyond interoperability and includes issues like question answering with multiple information sources of different quality, meaning negotiation, or knowledge management in large organizations. Starting with a focus on semantic interoperability makes the posing and solving of research problems more manageable, but the larger perspective on information integration already needs to guide our methodology.

\section{Acknowledgments}

Discussions with the members of MUSIL (http://musil.uni-muenster.de) and with too many colleagues to be named have greatly influenced and improved the ideas presented here. Funding from the University of Münster, the European ACE-GIS project (IST-2002-37724) and the meanings project in the German Ministry of Science Geotechnologies program is gratefully acknowledged.

\section{References}

1. EEA, CORINE Land Cover (Technical guide). European Environmental Agency, Commission of the European Community, 2000.

2. Bishr, Y., et al., Probing the Concept of Information Communities - A First Step Toward Semantic Interoperability, in Interoperating Geographic Information Systems (Proceedings of Interop'97), M.F. Goodchild, et al., Editors. 1999, Kluwer: pp. 55-71.

3. Sheth, A.P., Changing Focus on Interoperability in Information Systems: From System, Syntax, Structure to Semantics, in Interoperating Geographic Information Systems, M.F. Goodchild, et al., Editors. 1999, Kluwer: pp. 530.

4. Goguen, J.A. and R.M. Burstall, Institutions: abstract model theory for specification and programming. J. ACM, 1992. 39(1): pp. 95-146.

5. Goguen, J., Information Integration in Institutions (draft), in Memorial volume for Jon Barwise, L. Moss, Editor. (to appear).

http://www.cs.ucsd.edu/users/goguen/pps/ifi04.pdf. 
6. Montello, D. and S. Freundschuh, Cognition of Geographic Information, in A research agenda for geographic information science, R. McMaster and E. Usery, Editors. 2005, CRC Press: pp. 61-91.

7. Mark, D.M. and A.G. Turk. Landscape Categories in Yindjibarndi: Ontology, Environment, and Language. in Spatial Information Theory - Foundations of Geographic Information Science, COSIT 2003, Kartause Ittingen, Switzerland. 2003: Springer, Lecture Notes in Computer Science 2825: pp. 31-49.

8. Kuhn, W. Modeling the Semantics of Geographic Categories through Conceptual Integration. in Geographic Information Science - Second International Conference, GIScience 2002, Boulder, CO, USA, September 2002. 2002: Springer, Lecture Notes in Computer Science 2478: pp. 108-118.

9. Fonseca, F.T., et al., Using Ontologies for Integrated Geographic Information Systems. Transactions in GIS, 2002. 6(3): pp. 231-257.

10. Onsrud, H., et al., The Future of the Spatial Information Infrastructure, in A Research Agenda for Geographic Information Science, R.B. McMaster and E.L. Usery, Editors. 2005, CRC Press: pp. 225-255.

11. Harvey, F., et al., Semantic Interoperability: A Central Issue for Sharing Geographic Information. Annals of Regional Science, 1999. 33 (2)(Geospatial data sharing and standardization): pp. 213-232.

12. MacEachren, A.M., M. Gahegan, and W. Pike, Visualization for constructing and sharing geo-scientific concepts. PNAS, 2004. 101: Mapping Knowledge Domains: pp. 5279-5286.

13. Visser, U. and H. Stuckenschmidt. Interoperability in GIS - Enabling Technologies. in 5th AGILE Conference on Geographic Information Science. 2002. Palma de Mallorca, Spain: pp. 291-297.

14. Ogden, C.K. and I.A. Richards, The Meaning of Meaning. 1946: Harcourt, Brace \& World.

15. Timpf, S., Ontologies of Wayfinding. Networks and Spatial Economics, 2002(2): pp. 9-33.

16. Winter, S. and S. Nittel, Formal information modelling for standardisation in the spatial domain. International Journal of Geographical Information Science, 2003. 17(8): pp. 721-742.

17. Kuhn, W., Ontologies in support of activities in geographical space. International Journal of Geographical Information Science, 2001. 15(7): pp. 613631.

18. Gärdenfors, P., Conceptual Spaces - The Geometry of Thought. 2000: Bradford Books, MIT Press.

19. Hodges, W., Model Theory. 1993: Cambridge University Press.

20. Grüninger, M. Model-theoretic approaches to semantic integration (extended abstract). in Dagstuhl Seminar on Semantic Interoperability and Integration. 2004. Dagstuhl, Germany.

http://www.dagstuhl.de/files/Proceedings/04/04391/04391.GruningerMichae 13.Paper!.pdf.

21. Guarino, N., Formal Ontology and Information Systems, in Proc., 1st Int. Conf. on Formal Ontology in Information Systems, N. Guarino, Editor. 1998, IOS Press: pp. 3-15. 
22. Smith, B., Mereotopology: A Theory of Parts and Boundaries. Data and Knowledge Engineering, 1996. 20: pp. 287-303.

23. Gibson, J., The Theory of Affordances, in Perceiving, Acting, and Knowing Toward an Ecological Psychology, R. Shaw and J. Bransford, Editors. 1977, Lawrence Erlbaum Associates: pp. 67-82.

24. Rugg, R., M. Egenhofer, and W. Kuhn, Formalizing Behavior of Geographic Feature Types. Geographical Systems, 1997. 4(2): pp. 159-180.

25. Goguen, J.A., An Introduction to Algebraic Semiotics, with Application to User Interface Design, in Computation for Metaphors, Analogy and Agents, C. Nehaniv, Editor. 1999, Springer, Lecture Notes in Artificial Intelligence 1562: pp. 242-291.

26. Harnad, S., The Symbol Grounding Problem. Physica D, 1990. 42: pp. 335346.

27. Zlatev, J., Situated embodiment: Studies in the emergence of spatial meaning. 1997, Stockholm: Gotab.

28. Goguen, J.A., Ontology, Society, and Ontotheology, in Formal Ontology in Information Systems, Proceedings of the Third International Conference (FOIS 2004), A. Varzi and L. Vieu, Editors. 2004, IOS Press, 114: pp. 95103.

29. Fensel, D. and C. Bussler, The Web Service Modeling Framework WSMF. Electronic Commerce: Research and Applications, 2002(1): pp. 113-137.

30. Parnas, D.L., A Technique for Software Module Specification with Examples. Communications of the ACM, 1972. 15(5): pp. 1053-1058.

31. Cook, S. and J.D. Daniels, Designing Object Systems: Designing Object Oriented Modelling with Syntropy. 1994: Prentice Hall.

32. Buehler, K., ed. OpenGIS Reference Model. 2003, Open Geospatial Consortium (OGC).

33. Kottmann, C., Semantics and Information Communities, in The OpenGISTM Abstract Specification, C. Kottmann, Editor. Open GIS Consortium (OGC), 1999. http://www.opengeospatial.org/docs/99-114.pdf.

34. Riedemann, C. and C. Timm, Services for Data Integration. Data Science Journal, 2003. 2(26): pp. 90-99.

35. ISO, ISO 19107 - Spatial Schema. ISO TC 211, 2002.

36. Barr, M. and C. Wells, Category Theory for Computing Science. 1990: Prentice Hall.

37. Ehrig, H. and B. Mahr, Fundamentals of Algebraic Specification. 1985: Springer.

38. Woodcock, J. and M. Loomes, Software Engineering Mathematics. The SEI Series in Software Engineering. 1989: Addison Wesley.

39. Arpinar, I.B., et al., Geospatial Ontology Development and Semantic Analytics, ed. J.P. Wilson and A.S. Fotheringham. Handbook of Geographic Information Science. 2005 (in print): Blackwell Publishing.

40. Schade, S., et al. Comparing Approaches for Semantic Service Description and Matchmaking. in 3rd Int. Conf. on Ontologies, Data Bases, and Applications of Semantics for Large Scale Information Systems (ODBASE 2004). 2004. Larnaca, Cyprus: Springer, Lecture Notes in Computer Science 3291: pp. 1062-1079. 
41. Probst, F., et al. Connecting ISO and OGC Models to the Semantic Web (Extended Abstract). in Third International Conference on Geographic Information Science. 2004. Adelphi, MD, USA: pp. 181-184.

42. Kavouras, M. and M. Kokla, A Method for the Formalization and Integration of Geographic Categorizations. International Journal of Geographical Information Science, 2002. 16(5): pp. 439-453.

43. Frank, A., Ontology for spatio-temporal Databases, in Spatiotemporal Databases: The Chorochronos Approach, M.e.a. Koubarakis, Editor. 2003, Springer, 2520: pp. 9-77.

44. Lehnert, K., Su, Y., Langmuir, C.H., Sarbas, B., Nohl, U., A global geochemical database structure for rocks. Geochemistry, Geophysics, Geosystems, 2000. 1(May).

45. Worboys, M. and K. Hornsby, From Objects to Events: GEM, the Geospatial Event Mode, in Geographic Information Science. Third International Conference, GIScience 2004, M.J. Egenhofer, C. Freksa, and H.J. Miller, Editors. 2004, Springer, Lecture Notes in Computer Science 3234: pp. 327343.

46. Bennett, B. and M. Cristani, eds. Spatial Cognition and Computation: special issue on spatial vagueness, uncertainty and granularity. Spatial Cognition and Computation, ed. T. Cohn and S. Hirtle. Vol. 3(2-3). 2004, Springer.

47. Stell, J.G., Granularity in Change over Time, in Foundations of Geographic Information Science, M. Duckham, M. Goodchild, and M. Worboys, Editors. 2003, Taylor \& Francis: pp. 95-115.

48. Fonseca, F., et al., Semantic Granularity in Ontology-Driven Geographic Information Systems. Annals of Mathematics and Artificial Intelligence, 2002. 36(1-2): pp. 121-151.

49. Bishr, Y., Overcoming the Semantic and Other Barriers to GIS Interoperability. IJGIS, 1998. 12(4): pp. 299-314.

50. Sycara, K., et al., Dynamic Service Matchmaking Among Agents in Open Information Environments. SIGMOD Record, 1999. 28(1): pp. 47-53.

51. Klien, E., et al. An Architecture for Ontology-Based Discovery and Retrieval of Geographic Information. in 7th Conference on Geographic Information Science (AGILE 2004). 2004. Heraklion, Greece: Crete University Press: pp. 179-188.

52. Egenhofer, M. Toward the Semantic Geospatial Web. in 10th ACM International Symposium on Advances in Geographic Information Systems (ACMGIS). 2002. McLean, VA: pp. 1-4.

53. Lutz, M., Operation Ontologies for Semantic Discovery and Composition of Geoprocessing Services. Münster Semantic Interoperability Lab (MUSIL), 2005.

54. Riedemann, C., Naming Topological Operators at GIS User Interfaces. Münster Semantic Interoperability Lab (MUSIL), 2005.

55. Bernstein, A. and M. Klein. Towards High-Precision Service Retrieval. in The Semantic Web - First International Semantic Web Conference (ISWC 2002). 2002. Sardinia, Italy: pp. 84-101.

56. Iliffe, J.C., Datums and Map Projections. 2000. 
57. Vinoski, S., Web Services Interaction Models, Part 1: Current Practice. IEEE Internet Computing, 2002(May-June 2002): pp. 89-91.

58. Bernard, L., et al. Interoperability in GI Service Chains - The Way Forward. in 6th AGILE Conference on Geographic Information Science. 2003. Lyon, France.

59. Probst, F. and M. Lutz. Giving Meaning to GI Web Service Descriptions. in 2nd International Workshop on Web Services: Modeling, Architecture and Infrastructure (WSMAI-2004). 2004. Porto, Portugal.

60. Rodríguez, A. and M. Egenhofer, Comparing geospatial entity classes: an asymmetric and context-dependent similarity measure. International Journal of Geographical Information Science, 2004. 18(3): pp. 229-256.

61. Rodríguez, A. and M. Egenhofer. Putting Similarity Assessment into Context: Matching Functions with the User's Intended Operations. in Modeling and Using Context, CONTEXT'99. 1999. Trento, Italy: Springer-Verlag, Lecture Notes in Computer Science 1688: pp. 310-323.

62. Gärdenfors, P., How to Make the Semantic Web More Semantic, in Formal Ontology in Information Systems, Proceedings of the Third International Conference (FOIS 2004), A. Varzi and L. Vieu, Editors. 2004, IOS Press, 114: pp. 17-34.

63. Fabrikant, S.I. and B.P. Buttenfield, Formalizing Semantic Spaces for Information Access. Annals of the Association of American Geographers, 2001. 91: pp. 263-280.

64. Kuhn, W., Semantic Reference Systems. International Journal of Geographic Information Science (Guest Editorial), 2003. 17(5): pp. 405-409.

65. Chrisman, N., Exploring Geographic Information Systems. 2nd ed. 2002: Wiley.

66. Goodchild, M., Geographical data modeling. Computers and Geosciences, 1992. 18(4): pp. 401- 408.

67. Kuhn, W. and M. Raubal. Implementing Semantic Reference Systems. in AGILE 2003 - 6th AGILE Conference on Geographic Information Science. 2003. Lyon, France: Presses Polytechniques et Universitaires Romandes: pp. 63-72.

68. Berners-Lee, T., J. Hendler, and O. Lassila, The Semantic Web, in Scientific American, 2001. pp. 34-43.

69. Raubal, M., Formalizing Conceptual Spaces, in Formal Ontology in Information Systems, Proceedings of the Third International Conference (FOIS 2004), A. Varzi and L. Vieu, Editors. 2004, IOS Press, 114: pp. 153-164.

70. Doerr, M., J. Hunter, and C. Lagoze, Towards a Core Ontology for Information Integration. Journal of Digital information, 2003. 4(1).

71. Frank, A., Pragmatic Information Content: How to Measure the Information in a Route Description, in Foundations of Geographic Information Science, M. Duckham, M. Goodchild, and M. Worboys, Editors. 2003, Taylor \& Francis: pp. 47-68.

72. Oakley, T., Image Schema, in Handbook of Cognitive Linguistics, D. Geeraerts and H. Cuyckens, Editors. (in press), Oxford University Press. http://www.cwru.edu/artsci/engl/oakley/image_schema.pdf. 
73. Johnson, M., The Body in the Mind: The Bodily Basis of Meaning, Imagination, and Reason. 1987: The University of Chicago Press.

74. Lakoff, G., The Invariance Hypothesis: is abstract reason based on imageschemas? Cognitive Linguistics, 1990. 1(1): pp. 39-74.

75. Stevens, S.S., On the Theory of Measurement. Science, 1946. 103(2684): pp. 677-680.

76. Klein, F., Vergleichende Betrachtungen über neuere geometrische Forschungen. 1872: Verlag Andreas Deichert. 